\title{
Editorial
}

J. Synchrotron Rad. (1995). 2, 275

\section{A Perspective: JSR so far}

\author{
S. Samar Hasnain, ${ }^{a}$ John R. Helliwell ${ }^{b}$ and Hiromichi Kamitsubo ${ }^{c}$ \\ a Molecular Biophysics Group, CCLRC Daresbury Laboratory, Warrington WA4 4AD, UK, \\ ${ }^{b}$ Department of Chemistry, University of Manchester, Manchester M13 9PL, UK, and \\ cSpring-8, Jaeri-riken, Kamigori-cho, Hyogo-ken 678-12, Japan
}

This, the November issue, completes the second volume of the Journal of Synchrotron Radiation (JSR). It is timely then to look back over the year and share some of our thoughts with you on the progress of the journal. We set out to establish $J S R$ as the focus of the whole of the synchrotron radiation community and to accept only highquality papers covering sources, instrumentation, methods and applications. Our aim has been to provide fast and efficient publication to a high technical standard so that our rapidly expanding community is informed of the latest developments quickly and effectively. We sincerely believe that these objectives are being met.

The first two volumes have contained papers that have indeed covered all regions of the synchrotron radiation spectrum. In particular, we have seen papers covering sources, for example, on the performance of the first multi-GeV third-generation ESRF storage ring, through to, on the applications side, first reports of the highresolution spectroscopy that has become possible with the successful commissioning of the Advanced Light Source in Berkeley. During the last 12 months, we have also seen the successful operation of other new third-generation sources around the world. The news of the first beam in the Advanced Photon Source, the most powerful Xray source to date, flashed round the world in $1995[\mathrm{~J}$. Synchrotron Rad. (1995), 2, 162l. Also, the commissioning of the first instruments on ELETTRA in Trieste and the successful start of the Pohang light source in Korea and SRRC in Taiwan have happened smoothly. Thus, the excitement within the synchrotron radiation community and the growth of synchrotron radiation and its applications has continued.

It is our view that the generation and harnessing of synchrotron radiation involves common and overlapping instrumentation and methods in differing applications. Also, the importance of the cross-fertilization of ideas and de- velopments in one field of synchrotron radiation research, with another, cannot be overemphasized. Thus, from the outset, the journal has aimed to be the natural home for the reporting of synchrotron radiation research, irrespective of the synchrotron radiation technique or the region of the spectrum used. In this way, JSR has aimed to promote the rapid exchange of information across the whole synchrotron radiation community. That the journal has been well received can be judged by the steady increase in the number of papers submitted and in the placement of subscriptions. We are pleased that so many of you have taken part in this important but pioneering step, and have placed your faith and confidence in JSR.

As we enter into our third volume we feel confident that $J S R$ is here to stay as it is essential for the continued growth and stimulus of synchrotron radiation facilities, techniques and applications. But we approach a crucial period. This is the last complimentary issue of JSR. Thus, it is essential that we call upon you to ask your libraries and institutions to place subscriptions immediately. We would also like to remind you to consider the generous terms offered for personal subscriptions. For our part, we will continue to serve you and to work towards even higher technical and scientific standards. For this we again need your help and ask you always to consider $J S R$ as the first choice if you have results, methods or instrumental facilities that you would like the rest of the synchrotron radiation community to know about. We also welcome your comments and suggestions on the style and content of $J S R$, the journal of our rapidly expanding community.

We welcome a number of new members on the editorial board: G. E. Brown (Stanford), J. Kirz (Stony Brook), T.-N. Lee (Pohang), Y.-C. Liu (Taiwan), J. Penner-Hahn (Michigan), C. Riekel (Grenoble) and S. Suga (Osaka). This enlarged editorial board will provide improved geographical and scientific coverage. 